\title{
A GENERALIZATION OF THE AHLFORS-HEINS THEOREM 1
}

\author{
BY MATTS ESSÉN
}

Communicated by W. H. Fuchs, August 29, 1968

Let $D$ be the complex plane cut along the negative real axis. We are going to consider a function $u$ subharmonic in $D$. Let $M(r)$ $=\sup _{|z|=r} u(z)$ and $m(r)=\inf _{|z|=r} u(z)$. We also introduce, for $r>0, v(r)=\lim \sup _{z \rightarrow-r+i 0} u(z), \tilde{v}(r)=\lim \sup _{z \rightarrow-r-i 0} u(z)$ and $u(-r)$ $=\max (v(r), \tilde{v}(r))$. In the whole paper, $z=\mathrm{re}^{i \theta}$. Our main result is

THEOREM 1. Let $\lambda$ be a number in the interval $(0,1)$ and let $u(\not \equiv-\infty)$ be a function subharmonic in $D$ that satisfies

$$
u(-r)-\cos \pi \lambda u(r) \leqq 0 .
$$

Then either $\lim _{r \rightarrow \infty} r^{-\lambda} M(r)=\infty$ or

(A) there exists a number $\alpha$ such that

$$
\lim _{r \rightarrow \infty} r^{-\lambda} u\left(r e^{i \theta}\right)=\alpha \cos \lambda \theta, \quad|\theta|<\pi,
$$

except when $\theta$ belongs to a set of logarithmic capacity zero.

(B) Given $\theta_{0}, 0<\theta_{0}<\pi$, there exists an $r$-set $\Delta_{0}$ of finite logarithmic length such that (2) holds uniformly in $\left\{z|| \theta \mid \leqq \theta_{0}\right\}$ when $r$ is restricted to lie outside of $\Delta_{0}$.

REMARK. When $1 / 2<\lambda<1$, condition (1) is interpreted in the following way at points where $u(-r)=\infty$.

$$
\begin{aligned}
& \limsup _{z \rightarrow r}(u(x+i y)+u(-x+i y)) \leqq(1+\cos \pi \lambda) u(r), \\
& \limsup _{z \rightarrow r}(u(-x+i y)-\cos \pi \lambda u(x+i y)) \leqq 0 .
\end{aligned}
$$

Theorem 1 can be compared to the main result of Kjellberg [6].

THEOREM 2. Let $u$ be subharmonic in the complex plane and let $\lambda$ be a number in the interval $(0,1)$. If $m(r)-\cos \pi \lambda M(r) \leqq 0$, then the (possibly infinite) limit $\lim _{r \rightarrow \infty} r^{-\lambda} M(r)$ exists.

In order to clarify the connection between Theorem 1 and the Ahlfors-Heins theorem [1], we also state Theorem 1 in the following equivalent way.

\footnotetext{
${ }^{1}$ Research supported in part by the National Science Foundation.
} 
Theorem 3. Let $\lambda$ be a number in the interval $(0,1)$ and let $u$ be $a$ function subharmonic in $\{\operatorname{Re} z>0\}$. If for treal

$$
u(i t) \equiv \limsup _{z \rightarrow i t} u(z) \leqq \cos \pi \lambda u(|t|),
$$

then either $\lim \sup _{r \rightarrow \infty} r^{-2 \lambda} M(r)=\infty$ or $\lim _{z \rightarrow \infty} u(z) /\left(r^{2 \lambda} \cos 2 \lambda \theta\right)$ exists in the sense of $(\mathrm{A})$ and $(\mathrm{B})$.

If we choose $\lambda=1 / 2$, we obtain the Ahlfors-Heins theorem.

The proof of Theorem 1 is long and will appear elsewhere. In this announcement, we give an outline of the proof of Theorem 3 in the simpler case $0<\lambda<1 / 2$. In the proof, two lemmas (Lemmas 3 and 4) on convolution inequalities are used. These are stated at the end of the paper.

Proof of Theorem 3 in the Case $0<\lambda<1 / 2$. It is an unessential restriction to assume that $u$ is harmonic, bounded and has a negative upper bound in a neighborhood of the origin.

LEMMA 1. Under the assumptions of Theorem 3, either $\lim _{r \rightarrow \infty} r^{-2 \lambda} M(r)$ $=\infty$ or $\lim \sup _{r \rightarrow \infty} r^{-2 \lambda} u(r)<\infty$.

Proof of Lemma 1. We apply Poisson's formula for a semicircle (cf., e.g., Boas [2, 1.2.3]). Using (3), we deduce

$$
r^{-2 \lambda} u^{+}(r) \leqq \int_{0}^{R} t^{-2 \lambda} u^{+}(t) L(r, t) d t+\text { const. }(r / R)^{1-2 \lambda}\left(M(R) / R^{2 \lambda}\right),
$$

where

$$
L(r, t)=\frac{2 \cos \pi \lambda}{\pi} \cdot \frac{(t / r)^{2 \lambda} \cdot r}{t^{2}+r^{2}}
$$

and

$$
u^{+}=\max (u, 0) \text {. }
$$

Since $\int_{0}^{\infty} L(r, t) d t=1$, we obtain $\sup _{0<r<R} r^{-2 \lambda} u^{+}(r) \leqq$ const. $R^{-2 \lambda} M(R)$ from which Lemma 1 follows.

In the remaining part of the paper, we assume that the second alternative of Lemma 1 is valid. In particular, we have

$$
\liminf _{r \rightarrow \infty} r^{-2 \lambda} M(r)<\infty \text {. }
$$

Letting $R \rightarrow \infty$ in the formulas used in the proof of Lemma 1, we deduce 


$$
r^{-2 \lambda} u(r) \leqq \int_{0}^{\infty} L(r, t) t^{-2 \lambda} u(t) d t
$$

We define $\alpha=\lim \sup _{r \rightarrow \infty} r^{-2 \lambda} u(r)$ and $u_{1}(z)=u(z)-\alpha r^{2 \lambda} \cos 2 \lambda \theta$.

LEMMA 2. $\alpha$ is finite and $u_{1}$ is a nonpositive function on the positive real axis.

Proof. By the change of variables $r=e^{x}, t=e^{y}$ in (4), we obtain a convolution inequality

$$
\phi-\phi * L \leqq 0
$$

where

$$
L(x)=\frac{2 \cos \pi \lambda}{\pi} \frac{e^{(1-2 \lambda) x}}{e^{2 x}+1}
$$

and $\phi(x)=e^{-2 \lambda x} u\left(e^{x}\right)$. If $\alpha$ is finite, the lemma follows by applying Lemma 3 to $(\phi-\alpha)+$. The case $\alpha=-\infty$ is treated in a similar way.

From now on, we can assume that $\lim \sup _{r \rightarrow \infty} r^{-2 \lambda} u(r)=0$ and that $u$ is nonpositive on the positive real axis (if this is not true, replace $u$ by $\left.u_{1}\right)$. It follows from (3) that the function $\sim u(i t), t \in R$, is also. nonpositive. We define

$$
w(z)=\frac{r \cos \theta}{\pi} \int_{-\infty}^{\infty} \frac{u(i t)}{r^{2}-2 \operatorname{tr} \sin \theta+t^{2}} d t,
$$

the integral being absolutely convergent. Applying the PhragménLindelöf theorem (cf., e.g., Heins [5, p. 111]), we conclude that $w$ is a harmonic majorant of $u$ in $\{\operatorname{Re} z>0\}$. The nonnegative, superharmonic function $q$ is defined by $q=w-u$. Once more applying (3), we obtain

$$
w(r) \leqq \frac{2 r \cos \pi \lambda}{\pi} \int_{0}^{\infty} \frac{u(t)}{t^{2}+r^{2}} d t=\frac{2 r \cos \pi \lambda}{\pi} \int_{0}^{\infty} \frac{w(t)-q(t)}{t^{2}+r^{2}} d t .
$$

Since $q$ is nonnegative, the same change of variables as in the proof of Lemma 2 gives that the function $\psi$ defined by $\psi(x)=e^{-2 \lambda x} w\left(e^{x}\right)$, $x \in R$, is a solution of a convolution inequality. Applying Lemma 4, we obtain that $\lim _{x \rightarrow \infty} \psi(x)=\lim _{r \rightarrow \infty} r^{-2 \lambda} w(r)=0$ and that $\int_{0}^{\infty} t^{-1-2 \lambda} q(t) d t$ is convergent. It is now easy to prove that $\lim _{z \rightarrow \infty} w(z) /\left(r^{2 \lambda} \cos 2 \lambda \theta\right)$ $=0$ uniformly in each inner sector of $\{\operatorname{Re} z>0\}$. It remains for us to consider $q$.

We claim that $\lim _{z \rightarrow \infty} q(z) /\left(r^{2 \lambda} \cos 2 \lambda \theta\right)=0$ in the sense of (A) and (B). It is an unessential restriction to assume that $\lim _{z \rightarrow i t} q(z)=0$, 
$t \in R$ (if this is not true, replace $-q$ by $\max (-q,-x)$ ). Let the subharmonic function $h_{1}$ be $-q$ in the fourth quadrant and the least harmonic majorant of $-q$ in the first quadrant. Using repeated harmonic continuations and conformal mappings, we construct a function subharmonic in a half-plane which fulfills the assumptions of the Ahlfors-Heins theorem. The essential properties of the exceptional sets are not changed by conformal mappings, and going back to $h_{1}$, we obtain our result in the fourth quadrant. Interchanging the role of the quadrants in the previous construction, we obtain the existence of the limit in $\{\operatorname{Re} z>0\}$, and the proof of Theorem 3 in the case $0<\lambda<1 / 2$ is complete.

An alternative way of stating Theorem 3 is to use the concept of fine topology (cf. Doob [3] for references). It is worth mentioning that in the case $0<\lambda<1 / 2$, our assumptions imply that $u(z)$ has a finite fine limit almost everywhere on the imaginary axis. This property of $u$ follows immediately from Theorem 4.3 of Doob [3], applied to the nonpositive subharmonic function $u$ and the positive harmonic function $z \sim r^{2 \lambda} \cos 2 \lambda \theta, \operatorname{Re} z>0$.

Finally, we state the lemmas on convolution inequalities. They are variations on the result of Essén [4]. For simplicity, we only consider the kernel $L$ mentioned in the proof of Lemma 2, and study the convolution inequality

$$
\phi-\phi * L \leqq 0 .
$$

A solution of (5) is a locally integrable function $\phi$ such that $\phi * L$ converges absolutely and (5) is true.

Lemma 3. Let $\phi$ be a bounded solution of (5). If $\lim _{|x| \rightarrow \infty} \phi(x)=0$, then $\phi(x)=0$ a.e.

We define

$$
\begin{aligned}
\phi_{c}(x) & =\phi(x), & & \phi(x) \geqq-c, \\
& =-c, & & \phi(x)<-c .
\end{aligned}
$$

LeMma 4. Let $\phi$ be a nonpositive solution of (5). If $\lim \sup _{x \rightarrow \infty} \phi(x)$ $=0$, then $\phi-\phi * L \in L^{1}(0, \infty)$. If furthermore there exists a positive constant $c$ such that $\phi_{c}$ is slowly decreasing at infinity (cf. [7, Chapter IV (9b) ]), then $\lim _{x \rightarrow \infty} \phi(x)=0$.

\section{BIBLIOGRAPHY}

1. L. Ahlfors and M. Heins, Questions of regularity connected with the PhragmenLindelöf principle, Ann. of Math. (2) 50 (1949), 341-346.

2. R. P. Boas, Entire functions, Academic Press, New York, 1954. 
3. J. L. Doob, $A$ non-probabilistic proof of the relative Fatou theorem, Ann. Inst. Fourier (Grenoble) 9 (1959), 293-300.

4. M. Essén, Note on " $A$ theorem on the minimum modulus of entire functions" by Kjellberg, Math. Scand. 12 (1963), 12-14.

5. M. Heins, Selected topics in the classical theory of functions of a complex variable, Holt, New York, 1962.

6. B. Kjellberg, $A$ theorem on the minimum modulus of entire functions, Math. Scand. 12 (1963), 5-11.

7. D. V. Widder, The Laplace transform, Princeton Univ. Press, Princeton, N. J., 1946.

Royal Institute of Technology, Stockholm 70, Sweden and

UNIVERSITY OF ILLINOIS, URBANA, ILLINOIS 61801 